BULL. AUSTRAL. MATH. SOC.

\title{
THE MINIMAL NUMBER OF PERIODIC ORBITS OF PERIODS GUARANTEED IN SHARKOVSKII'S THEOREM
}

\author{
BAU-SEN Du
}

Let $f(x)$ be a continuous function from a compact real interval into itself with a periodic orbit of minimal period $m$, where $m$ is not an integral power of 2 . Then, by Sharkovskii's theorem, for every positive integer $n$ with $m \rightarrow n$ in the Sharkovskii ordering defined below, a lower bound on the number of periodic orbits of $f(x)$ with minimal period $n$ is 1 . Could we improve this lower bound from 1 to some larger number? In this paper, we give a complete answer to this question.

\section{Introduction}

Let $I$ be a compact real interval and let $f \in C^{0}(I, I)$. For any $x_{0}$ in $I$ and any positive integer $k$, we let $f^{k}\left(x_{0}\right)$ denote the $k$ th iterate of $x_{0}$ under $f$ and call $\left\{f^{k}\left(x_{0}\right) \mid k \geq 0\right\}$ the orbit of $x_{0}$ (under $f$ ). If $f^{m}\left(x_{0}\right)=x_{0}$ for some positive integer $m$, we call $x_{0}$ a periodic point of $f$ and call the cardinality of the orbit of $x_{0}$ (under $f$ ) the minimal period of $x_{0}$ and of the orbit (under $f$ ). If $f$ has a periodic orbit of a period $m$, must $f$ also have periodic orbits of periods $n \neq m$ ? In 1964, Sharkovski i [11] (see [1], [3], [7], [9], Received 23 August 1984.

Copyright Clearance Centre, Inc. Serial-fee code: 0004-9727/84 $\$$ A2.00 +0.00 . 
[12], [13], also) had given a complete answer to this question. Arrange the positive integers according as the following new order (called Sharkovskii ordering):

$$
\begin{aligned}
& 3 \quad \Delta \quad 5 \quad \Delta \quad 7 \quad \Delta \ldots \\
& \Delta \quad 2.3 \quad \Delta \quad 2.5 \quad \Delta \quad 2 \cdot 7 \quad \Delta \ldots \\
& \Delta 2^{n} \cdot 3 \quad \Delta 2^{n} \cdot 5 \quad \Delta \quad 2^{n} \cdot 7 \quad \Delta \ldots \\
& \vdots \quad \vdots \quad \vdots \\
& \Delta \quad \ldots \quad \Delta \quad 2^{3} \quad \Delta \quad 2^{2} \quad \Delta \quad 2 \quad \Delta \quad 1 .
\end{aligned}
$$

Sharkovskii's theorem says that any function $f \in C^{0}(I, I)$ with a periodic orbit of minimal period $m$, must also have at least one periodic orbit of minimal period $n$ precisely when $m \Delta n$ in the above Sharkovskii ordering. Therefore, for every positive integer $n$ with $m \Delta n$, the number $l$ is a lower bound on the number of distinct periodic orbits of $f$ with minimal period $n$. One question arises naturally: could we improve this lower bound from 1 to some larger number?

In 1976 Bowen and Franks [2] showed, among other things, that if $f \in C^{0}(I, I)$ has a periodic orbit of minimal period $n=2^{d} m$, where $m>I$ is odd, then there is a number $M_{n}$ (independent of $f$ ) such that, for all integers $k \geq M_{n}, f$ has at least $\left(2^{k / m}\right) /\left(2^{d} k\right)$ distinct periodic orbits of minimal period $2^{d} k$.

In 1979, Jonker [8] also obtained a similar result on a class of unimodal maps. If $c$ is an interior point of $I$, let $S_{c}$ denote the collection of all $f \in C^{0}(I, I)$ which has either one maximum or one minimum point at $c$, and is strictly monotone on each component of $I-\{c\}$ with $f(\partial I) \subset \partial I$. Jonker showed, among other things, that if $m, n$ are any two odd integers with $I<m<n$, and if $f \in S_{c}$ has a periodic orbit of minimal period $2^{k} m$, where $k \geq 0$ is any integer, then $f$ must also have at least $2^{(n-m) / 2}$ distinct periodic orbits of minimal period $2^{k} n$. 
In [6], a result along this line is also obtained. However, that result is only a partial one. In this paper we give a complete answer to that question.

In Section 2 we state our main results (Theorems 1, 2, and 3). In Section 3 we describe the method used to prove them. This method is the same as that used in [5] and [6]. The proofs of Theorems 1 and 2 will appear in Sections 4 and 5 . Theorem 3 then follows easily from theorems 1 and 2 .

\section{Statement of main results}

Let $\phi(m)$ be an integer-valued function defined on the set of all

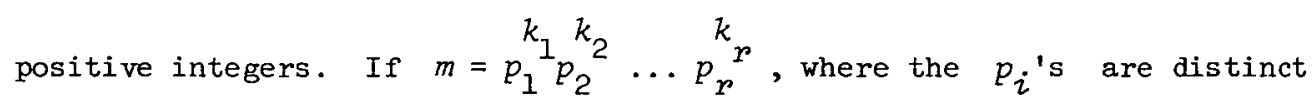
prime numbers, $r$ and $k_{i}{ }^{\prime} s$ are positive integers, we define

$$
\Phi(1, \phi)=\phi(1)
$$

and

$$
\begin{aligned}
\Phi(m, \phi)=\phi(m) & -\sum_{i=1}^{r} \phi\left(m / p_{i}\right)+\sum_{i_{1}<i_{2}} \phi\left(m /\left(p_{i_{1}} p_{i_{2}}\right)\right) \\
& -\sum_{i_{1}<i_{2}<i_{3}} \phi\left(m /\left(p_{i_{1}} p_{i_{2}} p_{i_{3}}\right)\right)+\ldots+(-1)^{r} \phi\left(m /\left(p_{1} p_{2} \ldots p_{r}\right)\right),
\end{aligned}
$$

where the summation $i_{1}<i_{2}<\ldots<i_{j}$ is taken over all $i_{1}, i_{2}, \ldots, i_{j}$ with $I \leq i_{1}<i_{2}<\ldots<i_{j} \leq r$. If, when considered as a sequence, $\langle\phi(m)\rangle$ is the Lucas sequence, that is if $\phi(1)=1, \phi(2)=3$, and $\phi(m+2)=\phi(m+l)+\phi(m)$ for all positive integers $m$, then, for simplicity, we denote $\Phi(m, \phi)$ as $\Phi_{1}(m)$. Note that if $f \in c^{0}(I, I)$ and if, for every positive integer $m, \phi(m)$ is the number of distinct solutions of the equation $f^{m}(x)=x$, then $\Phi(m, \phi)$ is, by the standard inclusion-exclusion argument, the number of periodic points of $f$ with minimal period $m$. Now we can state the following theorem.

THEOREM 1. Let $f:[1,3] \rightarrow[1,3]$ be defined by $f(x)=-2 x+5$ 
if $1 \leq x \leq 2$ and $f(x)=x-1$ if $2 \leq x \leq 3$. Then the following hold:

(a) for every positive integer $m$, if $a_{m}$ is the number of distinct solutions of the equation $f^{m}(x)=x$, then the sequence $\left\langle a_{m}\right\rangle$ is the Lucas sequence;

(b) for every positive integer $m$, f has exactly $\Phi_{1}(m) / m$ distinct periodic orbits of minimal period $m$;

(c) the sequence $\left\langle\Phi_{1}(m) / m\right\rangle$ is strictly increasing for $m \geq 6$ and $\lim _{n \rightarrow \infty}\left[\Phi_{1}(m+1) /(m+1)\right] /\left[\Phi_{1}(m) / m\right]=(1+\sqrt{5}) / 2 \ldots$

Fix any integer $n>1$ and let

$Q_{n}=\{(1, n+1)\} \cup\{(m, 2 n+2-m) \mid 2 \leq m \leq n\}$

$\cup\{(m, 2 n+1-m) \mid n+1 \leq m \leq 2 n\}$.

For all integers $i, j$, and $k$, with $1 \leq i, j \leq 2 n$ and $k \geq 1$, we define $b_{k, i, j, n}$ recursively as follows:

$$
b_{1, i, j, n}=\left\{\begin{array}{l}
1, \text { if }(i, j) \in Q_{n}, \\
0, \text { otherwise, }
\end{array}\right.
$$

and

$$
b_{k+1, i, j, n}=\left\{\begin{array}{ll}
b_{k, i, 2 n+1-j, n}+b_{k, i, n+1, n}, & \text { if } 1 \leq j \leq n-1, \\
b_{k, i, n, n}+b_{k, i, n+1, n}, & \text { if } j=n, \\
b_{k, i, 1, n}, & \text { if } j=n+1, \\
b_{k, i, 2 n+2-j, n}, & \text { if } n+2 \leq j \leq 2 n .
\end{array} .\right.
$$

We also define $c_{k, n}$ by letting

$$
c_{k, n}=\sum_{i=1}^{2 n} b_{k, i, i, n}+b_{k, n+1, n, n}+\sum_{i=n+2}^{2 n} b_{k, i, n+1, n} .
$$

Note that these sequences $\left\langle b_{k, i, j, n}\right\rangle$ and $\left\langle c_{k, n}\right\rangle$ have the following six properties. Some of these will be used later in the proofs of our main results. (Recall that $n>1$ is fixed.) 
(i) The sequence $\left\langle b_{k, I, n, n}\right\rangle$ is increasing, and for all integers $k \geq 2$, we have $b_{k, 1, n, n} \geq b_{k, n+1, n, n}$ and $b_{k, 1, i+1, n} \geq b_{k, 1, i, n}$ for all $1 \leq i \leq n-1$.

(ii) The sequences $\left\langle b_{k, 1, j, n}\right\rangle, 1 \leq j \leq n$, and $\left\langle b_{k, n+1, n, n}\right\rangle$ can also be obtained by the following recursive formulas:

$$
\begin{aligned}
& b_{1,1, j, n}=0,1 \leq j \leq n, \\
& b_{2,1, j, n}=1,1 \leq j \leq n, \\
& b_{1, n+1, n, n}=b_{2, n+1, n, n}=1, \\
& b_{1, n+1, j, n}=b_{2, n+1, j, n}=0,1 \leq j \leq n-1 .
\end{aligned}
$$

For $i=1$ or $n+1$, and $k \geq 1$,

$$
\begin{aligned}
& b_{k+2, i, n, n}=b_{k, i, 1, n}+b_{k+1, i, n, n}, \\
& b_{k+2, i, j, n}=b_{k, i, 1, n}+b_{k, i, j+1, n}, \quad 1 \leq j \leq n-1 .
\end{aligned}
$$

(iii) For every positive integer $k, c_{k+2 n-2, n}$ can also be obtained by the following formulas:

$$
\begin{aligned}
c_{k+2 n-2, n} & =b_{k+2 n-2, n+1, n, n}+2 \sum_{j=1}^{n} b_{k+2 n-2 j, 1, j, n} \\
& =b_{k+2 n-2, n+1, n, n}+2 n b_{k, 1, n, n}+\sum_{i=2}^{n}\left(2^{i}-2\right) b_{k, 1, n+1-i, n} .
\end{aligned}
$$

The first identity also holds for all integers $k$ with $-2 n+3 \leq k \leq 0$ provided we define $b_{k, 1, j, n}=0$ for all $-2 n+3 \leq k \leq 0$ and $1 \leq j \leq n$.

(iv) For all integers $k$ with $1 \leq k \leq 2 n, c_{2 k, n}=2^{k+1}-1$.

(v) For all integers $k$ with $n+1 \leq k \leq 3 n$, $c_{2 k+1, n}=2 c_{2 k+1, n+1}-1$.

(vi) Since, for every positive integer $k \geq 2 n+1$,

$$
b_{k, 1, n, n}=b_{k-1,1, n, n}+\sum_{i=2}^{2 n}(-1)^{i} b_{k-i, 1, n, n},
$$


there exist $2 n+1$ nonzero constants $\alpha_{j}$ 's such that $b_{k, 1, n, n}=\sum_{j=1}^{2 n+1} \alpha_{j} x_{j}^{k}$ for all positive integers $k$, where $\left\{x_{j} \mid 1 \leq j \leq 2 n+1\right\}$ is the set of all zeros (including complex zeros) of the polynomial $x^{2 n+1}-2 x^{2 n-1}-1$.

For all positive integers $k, m, n$, with $n>1$, we let $\phi_{n}(k)=c_{k, n}$ and let $\Phi_{n}(m)=\Phi\left(m, \phi_{n}\right)$, where $\Phi$ is defined as above. Now we can state the following theorem.

THEOREM 2. For every integer $n>1$, let

$$
f_{n}:[1,2 n+1] \rightarrow[1,2 n+1]
$$

be the continuous fronction with the following six properties:
(1) $f_{n}(1)=n+1$,
(2) $f_{n}(2)=2 n+1$,
(3) $f_{n}(n+1)=n+2$,
(4) $f_{n}(n+2)=n$,
(5) $f_{n}(2 n+1)=1$, and
(6) $f_{n}$ is Zinear on each component of the complement of the set $\{2, n+1, n+2\}$ in $[1,2 n+1]$.

Then the following hold:

(a) for every positive integer $k$, the equation $f_{n}^{k}(x)=x$ has exactly $c_{k, n}$ distinct solutions;

(b) for every positive integer $m, f_{n}(x)$ has exactly $\Phi_{n}(m) / m$ distinct periodic orbits of minimal period $m$;

(c) $\lim _{m \rightarrow \infty}\left(\log \left[\Phi_{n}(m) / m\right]\right) / m=\lambda_{n}$, where $\lambda_{n}$ is the (wanique) positive (and the largest in absolute value) zero of the polynomial $x^{2 n+1}-2 x^{2 n-1}-1$. 
From Theorems 1 and 2 above and Theorem 2 of [12, p. 243], we easily obtain the following result.

THEOREM 3. Assume that $f \in C^{0}(I, I)$ has a periodic orbit of minimal period $s=2^{k}(2 n+1)$, where $n \geq 1$ and $k \geq 0$, and no periodic orbits of minimal period $r$ with $r \Delta s$ in the Sharkovskii ordering. Then for every positive integer $t$ with $s \Delta t$ in the Sharkovskii ordering, $f$ has at least $\Phi_{n}\left(t / 2^{k}\right) /\left(t / 2^{k}\right)$ (sharp) distinct periodic orbits of minimal period $t$.

REMARK 1 . We call attention to the fact that there exist continuous functions from $I$ into $I$ with exactly one periodic orbit of minimal period $2^{i}$ for every positive integer $i$ (and two fixed points), but no other periods (see [10]).

REMARK 2. With the help of Theorem 2 of $[12, p .243]$ on the distribution along the real line of points in a periodic orbit of odd period $n>1$, when there are no periodic orbits of odd period $m$ with $1<m<n$, our results give a new proof of Sharkovskii's theorem.

REMARK 3. Table 1 (see p. 96) lists the first 31 values of $\Phi_{n}(m) / m$ for $1 \leq n \leq 5$. It seems that, for all positive integers $n$ and $m$, we have

$$
\Phi_{n}(2 m+1) /(2 m+1)=2^{m-n} \text { for } n \leq m \leq 3 n+1 \text {, }
$$

and

$$
\Phi_{n}(2 m+1) /(2 m+1)>2^{m-n} \text { for } m>3 n+1 \text {. }
$$

REMARK 4. For all positive integers $k$ and $m$, let $\psi(k)=2^{k}$ and $\Psi(m)=\Phi(m, \psi)$, where $\Phi$ is defined as in Section 2. It is obvious that $\Psi(m) / m$ is the number of distinct periodic orbits of minimal period $m$ for, say, the mapping $g(x)=4 x(1-x)$ from $[0,1]$ onto itself. Since, for all positive integers $k$ and $n$ with $1 \leq k \leq 2 n, c_{2 k, n}=2^{k+1}-1$ $c_{1, n}=1$, we obtain that $\Phi_{n}(2 k+2) /(2 k+2)=\Psi(k+1) /(k+1)$ for all 
TABLE 1

\begin{tabular}{|c|c|c|c|c|c|c|}
\hline$m$ & $\Phi_{1}(m) / m$ & $\Phi_{2}(m) / m$ & $\Phi_{3}(m) / m$ & $\Phi_{4}(m) / m$ & $\Phi_{5}(m) / m$ & $\Psi(m) / m$ \\
\hline 1 & 1 & 1 & 1 & 1 & 1 & 2 \\
\hline 2 & 1 & 1 & 1 & 1 & 1 & 1 \\
\hline 3 & 1 & 0 & 0 & 0 & 0 & 2 \\
\hline 4 & 1 & 1 & 1 & 1 & 1 & 3 \\
\hline 5 & 2 & 1 & 0 & 0 & 0 & 6 \\
\hline 6 & 2 & 2 & 2 & 2 & 2 & 9 \\
\hline 7 & 4 & 2 & 1 & 0 & 0 & 18 \\
\hline 8 & 5 & 3 & 3 & 3 & 3 & 30 \\
\hline 9 & 8 & 4 & 2 & 1 & 0 & 56 \\
\hline 10 & 11 & 6 & 6 & 6 & 6 & 99 \\
\hline 11 & 18 & 8 & 4 & 2 & 1 & 186 \\
\hline 12 & 25 & 11 & 9 & 9 & 9 & 335 \\
\hline 13 & 40 & 16 & 8 & 4 & 2 & 630 \\
\hline 14 & 58 & 23 & 18 & 18 & 18 & 1161 \\
\hline 15 & 90 & 32 & 16 & 8 & 4 & 2182 \\
\hline 16 & 135 & 46 & 32 & 30 & 30 & 4080 \\
\hline 17 & 210 & 66 & 32 & 16 & 8 & 7710 \\
\hline 18 & 316 & 94 & 61 & 56 & 56 & 14560 \\
\hline 19 & 492 & 136 & 64 & 32 & 16 & 27594 \\
\hline 20 & 750 & 195 & 115 & 101 & 99 & 52377 \\
\hline 21 & 1164 & 282 & 128 & 64 & 32 & 99858 \\
\hline 22 & 1791 & 408 & 224 & 191 & 186 & 190557 \\
\hline 23 & 2786 & 592 & 258 & 128 & 64 & 364722 \\
\hline 24 & 4305 & 856 & 431 & 351 & 337 & 698870 \\
\hline 25 & 6710 & 1248 & 520 & 256 & 128 & 1342176 \\
\hline 26 & 10420 & 1814 & 850 & 668 & 635 & 2580795 \\
\hline 27 & 16264 & 2646 & 1050 & 512 & 256 & 4971008 \\
\hline 28 & 25350 & 3858 & 1673 & 1257 & 1177 & 9586395 \\
\hline 29 & 39650 & 5644 & 2128 & 1026 & 512 & 18512790 \\
\hline 30 & 61967 & 8246 & 3328 & 2402 & 2220 & 35790267 \\
\hline 31 & 97108 & 12088 & 4320 & 2056 & 1024 & 69273666 \\
\hline
\end{tabular}


$1 \leq k \leq 2 n$. It seems that $\Phi_{n}(2 k+2) /(2 k+2)>\Psi(k+1) /(k+1)$ for all

$k>2 n$. But note that

$\lim _{k \rightarrow \infty}\left(\log \left[\Phi_{n}(2 k+2) /(2 k+2)\right]\right) /(2 k+2)$

$$
=\log \lambda_{n}>\frac{1}{2} \log 2=\frac{1}{2} \lim _{k \rightarrow \infty}(\log [\Psi(k+1)] /(k+1)) /(k+1),
$$

where $\lambda_{n}$ is the unique positive zero of the polynomial

$x^{2 n+1}-2 x^{2 n-1}-1$.

\section{Symbolic representation for continuous piecewise linear functions}

In this section we describe a method. This method was first introduced in [4], and then generalized in [5] to construct, for every positive integer $n$, a continuous piecewise linear function from $[0,1]$ into itself which has a periodic orbit of minimal period 3 , but with the property that almost all (in the sense of Lebesgue) points of $[0,1]$ are eventually periodic of minimal period $n$ with the periodic orbit the same as the orbit of a fixed known period $n$ point. The same method was also used in [6] to give a new proof of a result of Block et al [1] on the topological entropy of interval maps. In this paper we will use this method to prove our main results.

Throughout this section, let $g$ be a continuous piecewise linear function from the interval $[c, d]$ into itself. We call the set $\left\{\left(x_{i}, y_{i}\right) \mid i=1,2, \ldots, k\right\}$ a set of nodes for (the graph of) $y=g(x)$ if the following three conditions hold:

(1) $k \geq 2$,

(2) $x_{1}=c, x_{k}=d, x_{1}<x_{2}<\ldots<x_{k}$, and

(3) $g$ is linear on $\left[x_{i}, x_{i+1}\right]$ for all $1 \leq i \leq k-1$ and $y_{i}=g\left(\dot{x}_{i}\right)$ for all $1 \leq i \leq k$.

For any such set, we will use its $y$-coordinates $y_{1}, y_{2}, \ldots, y_{k}$ to represent the graph of $y=g(x)$ and call $y_{1} y_{2} \cdots y_{k}$ (in that order) a (symbolic) representation for (the graph of) $y=g(x)$. For 
$1 \leq i<j \leq k$, we will call $y_{i} y_{i+1} \cdots y_{j}$ the representation for $y=g(x)$ on $\left[x_{i}, x_{j}\right]$ obtained by restricting $y_{1} y_{2} \ldots y_{k}$ to $\left[x_{i}, x_{j}\right]$. For convenience, we will also call every $y_{i}$ in $y_{1} y_{2} \cdots y_{k}$ a node. If $y_{i}=y_{i+1}$ for some $i$ (that is, $g$ is constant on $\left[x_{i}, x_{i+1}\right]$, we will simply write $y_{1} \ldots y_{i} y_{i+2} \cdots y_{k}$ instead of $y_{1} \cdots y_{i} y_{i+1} y_{i+2} \cdots y_{k}$. Therefore, every two consecutive nodes in a (symbolic) representation are distinct.

Now assume that $\left\{\left(x_{i}, y_{i}\right\} \mid i=1,2, \ldots, k\right\}$ is a set of nodes for $y=g(x)$ and $a_{1} a_{2} \ldots a_{p}$ is a representation for $y=g(x)$ with $\left\{a_{1}, a_{2}, \ldots, a_{p}\right\} \subset\left\{y_{1}, y_{2}, \ldots, y_{k}\right\}$ and $a_{i} \neq a_{i+1}$ for all $1 \leq i \leq p-1$. If $\left\{y_{1}, y_{2}, \ldots, y_{k}\right\} \subset\left\{x_{1}, x_{2}, \ldots, x_{k}\right\}$, then there is an easy way to obtain a representation for $y=g^{2}(x)$ from the one $a_{1} a_{2} \ldots a_{r}$ for $y=g(x)$. The procedure is as follows. First, for any two distinct real numbers $u$ and $v$, let $[u: v]$ denote the closed interval with endpoints $u$ and $v$. Then let $b_{i, 1} b_{i, 2} \cdots b_{i, t_{i}}$ be the representation for $y=g(x)$ on $\left[a_{i}: a_{i+1}\right]$ which is obtained by restricting $a_{1} a_{2} \ldots a_{p}$ to $\left[a_{i}: a_{i+1}\right]$. We use the following notation to indicate this fact: $a_{i} a_{i+1} \rightarrow b_{i, 1} b_{i, 2} \cdots b_{i, t_{i}}$ (under $g$ ) if $a_{i}<a_{i+1}$, or $a_{i} a_{i+1} \rightarrow b_{i, t_{i}} \ldots b_{i, 2} b_{i, 1}$ (under $g$ ) if $a_{i}>a_{i+1}$. The above representation on $\left[a_{i}: a_{i+1}\right]$ exists since $\left\{a_{1}, a_{2}, \ldots, a_{p}\right\} \subset\left\{x_{1}, x_{2}, \ldots, x_{k}\right\}$. Finally, if $a_{i}<a_{i+1}$, let $z_{i, j}=b_{i, j ;}$ for all $1 \leq j \leq t_{i}$. If $a_{i}>a_{i+1}$, let $z_{i, j}=b_{i, t_{i}+1-j}$ for all $1 \leq j \leq t_{i}$. It is easy to see that $z_{i, t_{i}}=z_{i+1,1}$ for all $1 \leq i \leq r-1$. So, if we define

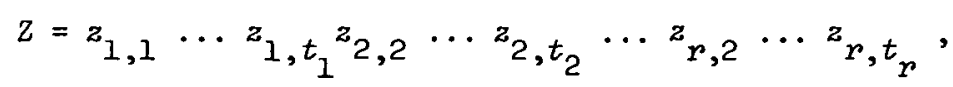

then it is obvious that $z$ is a representation for $y=g^{2}(x)$. It is 
also obvious that the above procedure can be applied to the representation $z$ for $y=g^{2}(x)$ to obtain one for $y=g^{3}(x)$, and so on.

\section{Proof of Theorem 1}

In this section we let $f(x)$ denote the map as defined in Theorem 1 , that is $f(x)=-2 x+5$ if $1 \leq x \leq 2$, and $f(x)=x-1$ if $2 \leq x \leq 3$. The proof of part (a) of Theorem 1 will follow from two easy lemmas.

LEMMA 4. Under $f$, we have

$$
\begin{aligned}
& 13 \rightarrow 312, \quad 31 \rightarrow 213, \\
& 12 \rightarrow 31, \quad 21 \rightarrow 13 .
\end{aligned}
$$

In the following when we say the representation for $y=f^{k}(x)$, we mean the representation obtained, following the procedure as described in Section 3, by applying Lemma 4 to the representation 312 for $y=f(x)$ successively until we get to the one for $y=f^{k}(x)$.

For every positive integer $k$, let $u_{1, k}\left(u_{2, k}\right.$ respectively $)$ denote the number of $13^{\prime} \mathrm{s}$ and $31^{\prime} \mathrm{s}$ in the representation for $y=f^{k}(x)$ whose corresponding $x$-coordinates are $\leq(\geq$ respectively) 2 . We also let $v_{1, k}\left(v_{2, k}\right.$ respectively $)$ denote the number of 12 's and 21 's in the representation for $y=f^{k}(x)$ whose corresponding $x$-coordinates are $\leq$ ( $\geq$ respectively) 2 . It is clear that $u_{1,1}=v_{2,1}=1$ and $u_{2, I}=v_{1,1}=0$. Now from Lemma 4 , we have

LEMMA 5. For every positive integer $k$ and integers $i=1,2$, $u_{i, k+1}=u_{i, k}+v_{i, k}$ and $v_{i, k+1}=u_{i, k}$. Furthermore, if $w_{k}=u_{1, k}+v_{1, k}+u_{2, k}$, then $w_{1}=1, w_{2}=3$, and $w_{k+2}=w_{k+1}+w_{k}$. That is, $\left\langle w_{k}\right\rangle$ is the Lucas sequence.

Since, for every positive integer $k$, the number of distinct solutions of the equation $f^{k}(x)=x$ equals $w_{k}$, part $(a)$ of Theorem 1 follows from Lemma 5. Part (b) follows from the standard inclusionexclusion argument. As for part (c), we note that, for every positive 
integer $k$,

$$
a_{k+2}=\sum_{i=1}^{k} a_{i}+3 .
$$

So, for $k \geq 6$,

$$
\begin{aligned}
(k+2) \Phi_{1}(k+3) & >(k+2)\left(a_{k+3}-a_{[(k+3) / 2]+1}\right) \\
& >(k+3)\left(a_{k+2}+a_{[(k+3) / 2]+1}\right) \\
& >(k+3) \Phi_{1}(k+2),
\end{aligned}
$$

where $[(k+3) / 2]$ is the largest integer less than or equal to $(k+3) / 2$. The proof of the other statement of part (c) is easy and omitted. This completes the proof of Theorem 1 .

\section{Proof of Theorem 2}

In this section we fix any integer $n>I$ and let $f_{n}(x)$ denote the map as defined in Theorem 2. For convenience, we also let $S_{n}$ denote the set of all these $4 n$ symbolic pairs: $i(i+1),(i+1) i, 1 \leq i \leq n-1$; $n(n+2),(n+2) n,(n+1)(2 n+1),(2 n+1)(n+1), j(j+1),(j+1) j, n+2 \leq j \leq 2 n$.

The following lemma is easy.

LEMMA 6. Under $f_{n}$, we have

$$
\begin{aligned}
n(n+2) & \rightarrow(n+3)(n+2) n, \quad(n+2) n \rightarrow n(n+2)(n+3), \\
(n+1)(2 n+1) & \rightarrow(n+2) n(n-1)(n-2) \ldots 321, \\
(2 n+1)(n+1) & \rightarrow 123 \ldots(n-2)(n-1) n(n+2),
\end{aligned}
$$

and $u v \rightarrow f_{n}(u) f_{n}(v)$ for every $u v$ in

$$
S_{n}-\{n(n+2),(n+2) n,(n+1)(2 n+1),(2 n+1)(n+1)\} \text {. }
$$

In the following when we say the representation for $y=f_{n}^{k}(x)$, we mean the representation obtained, following the procedure as described in Section 3, by applying Lemma 6 to the representation

$$
(n+1)(2 n+1)(2 n)(2 n-1) \ldots(n+2) n(n-1)(n-2) \ldots 321
$$


for $y=f_{n}(x)$ successively until we get to the one for $y=f_{n}^{k}(x)$.

For every positive integer $k$ and all integers $i, j$ with $1 \leq i, j \leq 2 n$, let $b_{k, i, j, n}$ denote the number of $u v^{\prime} s$ and $v u^{\prime} s$ in the representation for $y=f_{n}^{k}(x)$ whose corresponding $x$-coordinates are in $[i, i+1]$, where $u v=j(j+1)$ if $1 \leq j \leq n-1$ or $n+2 \leq j \leq 2 n$, $u v=n(n+2)$ if $j=n$, and $u v=(n+1)(2 n+1)$ if $j=n+1$. It is obvious that $b_{1,1, n+1, n}=1, b_{1, i, 2 n+2-i, n}=1$ if $2 \leq i \leq n$, $b_{1, i, 2 n+1-i, n}=1$ if $n+1 \leq i \leq 2 n$, and $b_{1, i, j, n}=0$ elsewhere. From Lemma 6 , we see that the sequences $\left\langle b_{k, i, j, n}\right\rangle$ are exactly the same as those defined in Section 2 .

Since

$$
c_{k, n}=\sum_{i=1}^{2 n} b_{k, i, i, n}+b_{k, n+1, n, n}+\sum_{i=n+2}^{2 n} b_{k, i, n+1, n} \text {, }
$$

it is clear that $c_{k, n}$ is the number of intersection points of the graph of $y=f_{n}^{k}(x)$ with the diagonal $y=x$. This proves part $(a)$ of Theorem 2. Part (b) follows from the standard inclusion-exclusion argument. As for part $(c)$, we note that there exist $2 n+1$ nonzero constants $\alpha_{j}$ 's such that

$$
b_{k, 1, n, n}=\sum_{j=1}^{2 n+1} \alpha_{j} x_{j}^{k}
$$

for all positive integers $k$, where $\left\{x_{j} \mid 1 \leq j \leq 2 n+1\right\}$ is the set of all zeros (including complex zeros) of the polynomial $x^{2 n+1}-2 x^{2 n-1}-1$. Since $c_{k+2 n-2, n}$ can also be expressed as

$$
b_{k+2 n-2, n+1, n, n}+2 n b_{k, 1, n, n}+\sum_{i=2}^{n}\left(2^{i}-2\right) b_{k, 1, n+1-i, n},
$$

part (c) follows from property (i) of the sequences $\left\langle b_{k, i, j, n}\right\rangle$ stated in Section 2. This completes the proof of Theorem 2 . 


\section{References}

[1] L. Block, J. Guckenheimer, M. Misiurewicz and L.-S. Young, "Periodic points and topological entropy of one dimensional maps", 18-34 (Lecture Notes in Mathematics, 819. Springer-Verlag, Berlin, Heidelberg, New York, 1980).

[2] R. Bowen and J. Franks, "The periodic points of maps of the disk and the interval", Topology 15 (1976), 337-342.

[3] U. Burkart, "Interval mapping graphs and periodic points of continuous functions", J. Combin. Theory Ser. B 32 (1982), 57-68.

[4] Bau-Sen Du, "Almost all points are eventually periodic with minimal period 3 ", Bulz. Inst. Math. Acad. Sinica 12 (1984), 405-411.

[5] Bau-Sen Du, "Almost all points are eventually periodic with same periodic orbit" (Preprint, Academia Sinica, Taiwan, Republic of China, 1984).

[6] Bau-Sen Du, "The periodic points and topological entropy of interval maps" (Preprint, Academia Sinica, Taiwan, Republic of China, 1984).

[7] C. W. Ho and C. Morris, "A graph theoretic proof of Sharkovsky's theorem on the periodic points of continuous functions", Pacific J. Math. 96 (1981), 361-370.

[8] L. Jonker, "Periodic points and kneading invariants", Proc. London Math. Soc. (3) 39 (1979), 428-450.

[9] Tien-Yien Li and James A. Yorke, "Period three implies chaos", Amer. Math. Monthly 82 (1975), 985-992.

[10] M. Misiurewicz, "Structure of mappings of an interval with zero entropy", Inst. Hautes Études Sci. Publ. Math. 53 (1981), 5-16.

[11] A.N. Sharkovskii, "Coexistence of cycles of a continuous map of a line into itself", Ukrain. Mat. Z. 16 (1964), 61-71.

[12] P. Stefan, "A theorem of Sharkovsky on the existence of periodic orbits of continuous endomorphisms of the real line", Comm. Math. Phys. 54 (1977), 237-248. 
[13] P.D. Straffin, Jr., "Periodic points of continuous functions", Math. Mag. 51 (1978), 99-105.

Institute of Mathematics,

Academia Sinica,

Nankang,

Taipei,

Taiwan 115,

Republic of China. 